\title{
Asymmetric Janus adhesive tape prepared by interfacial hydrosilylation for wet/dry amphibious adhesion
}

\author{
Xizi Wan ${ }^{1,2}$, Zhen Gu ${ }^{1,3,4}$, Feilong Zhang ${ }^{2}$, Dezhao Hao ${ }^{1,2}$, Xi Liu' ${ }^{1,2}$, Bing Dai ${ }^{1,2}$, Yongyang Song ${ }^{1,2}$ and Shutao Wang ${ }^{1,2,4}$
}

\begin{abstract}
Janus films with asymmetric properties on opposite sides have been widely used to facilitate energy storage, ion transport, nanofiltration, and responsive bending. However, studies on Janus films rarely involve controlling surface adhesion, either dry or wet adhesion. Herein, we report Janus adhesive tape with an asymmetrically crosslinked polydimethylsiloxane (PDMS) network prepared through an interfacial hydrosilylation strategy, realizing wet/dry amphibious adhesion on various solid surfaces. The lightly crosslinked side of the Janus adhesive tape acts as an adhesive layer with high adhesion, and the highly crosslinked side functions as a supporting layer with high mechanical strength. This Janus adhesive tape with good adhesion and mechanical properties can be dyed different colors and can act as an underwater adhesive and a skin adhesive for wearable electronic devices. This study provides a promising design model for next-generation adhesive materials and related applications.
\end{abstract}

\section{Introduction}

Janus films with asymmetric features on opposite sides have been emerging as important functional materials that strongly affect various applications in energy storage ${ }^{1}$, ion transport $^{2,3}$, nanofiltration ${ }^{4}$, and responsive bending ${ }^{5-7}$. Many unique methods have been developed to prepare a series of functional Janus films with asymmetric properties such as wettability, surface charge, and morphology ${ }^{8,9}$. For example, hydrophilic-hydrophobic Janus films were fabricated by electrospinning as materials for excess biofluid removal $^{10}$ or as battery separators ${ }^{1}$. Electrostatic heterostructure Janus films were fabricated by spin-coating and microphase separation for ion rectification ${ }^{3,11,12}$. Hydrogel-elastomer/organogel Janus films were fabricated by interfacial polymerization for mechanical robustness, antifouling $^{13,14}$, or responsive bonding ${ }^{5,15}$. However,

\footnotetext{
Correspondence: Shutao Wang (stwang@mail.ipc.ac.cn)

${ }^{1}$ CAS Key Laboratory of Bioinspired Materials and Interfacial Science, CAS

Center for Excellence in Nanoscience, Technical Institute of Physics and

Chemistry, Chinese Academy of Sciences, Beijing 100190, P. R. China

${ }^{2}$ University of Chinese Academy of Sciences, Beijing 100049, P. R. China

Full list of author information is available at the end of the article.

These authors contributed equally: Xizi Wan, Zhen Gu
}

studies on Janus films rarely involve controlling surface adhesion, either dry or wet adhesion.

Many unique adhesion phenomena in nature, such as dry adhesion of geckos ${ }^{16-19}$ and wet adhesion of mussels $^{20-23}$, barnacles ${ }^{24}$, and octopi ${ }^{25}$, have inspired the rational design of functional adhesive materials. Geckoinspired dry adhesion ${ }^{26}$ and octopus-inspired wet adhesion $^{25,27}$ are often based on elaborate replicating templates with unique structures. Mussel- and barnacleinspired wet adhesion is based on molecular design, such as graft ${ }^{28}$ or block ${ }^{29,30}$ copolymerization. By synergistically combining the salient elements of both gecko and mussel adhesives, PDMS micro-/nanopillar arrays coated with a thin layer of synthetic catechol-based polymers have been fabricated to achieve adhesion in both dry and wet environments $^{31,32}$. However, the elaborate preconstruction of surface micro-/nanostructures or the introduction of catechol groups into adhesive polymers greatly restricts the practical applications of these functional adhesives. It is still a great challenge to develop wet/dry amphibious adhesive materials without laborious replicating structure preparation and molecular design.

\section{(c) The Author(s) 2019}

(c) Open Access This article is licensed under a Creative Commons Attribution 4.0 International License, which permits use, sharing, adaptation, distribution and reproduction cc) in any medium or format, as long as you give appropriate credit to the original author(s) and the source, provide a link to the Creative Commons license, and indicate if changes were made. The images or other third party material in this article are included in the article's Creative Commons license, unless indicated otherwise in a credit line to the material. If material is not included in the article's Creative Commons license and your intended use is not permitted by statutory regulation or exceeds the permitted use, you will need to obtain permission directly from the copyright holder. To view a copy of this license, visit http://creativecommons.org/licenses/by/4.0/. 


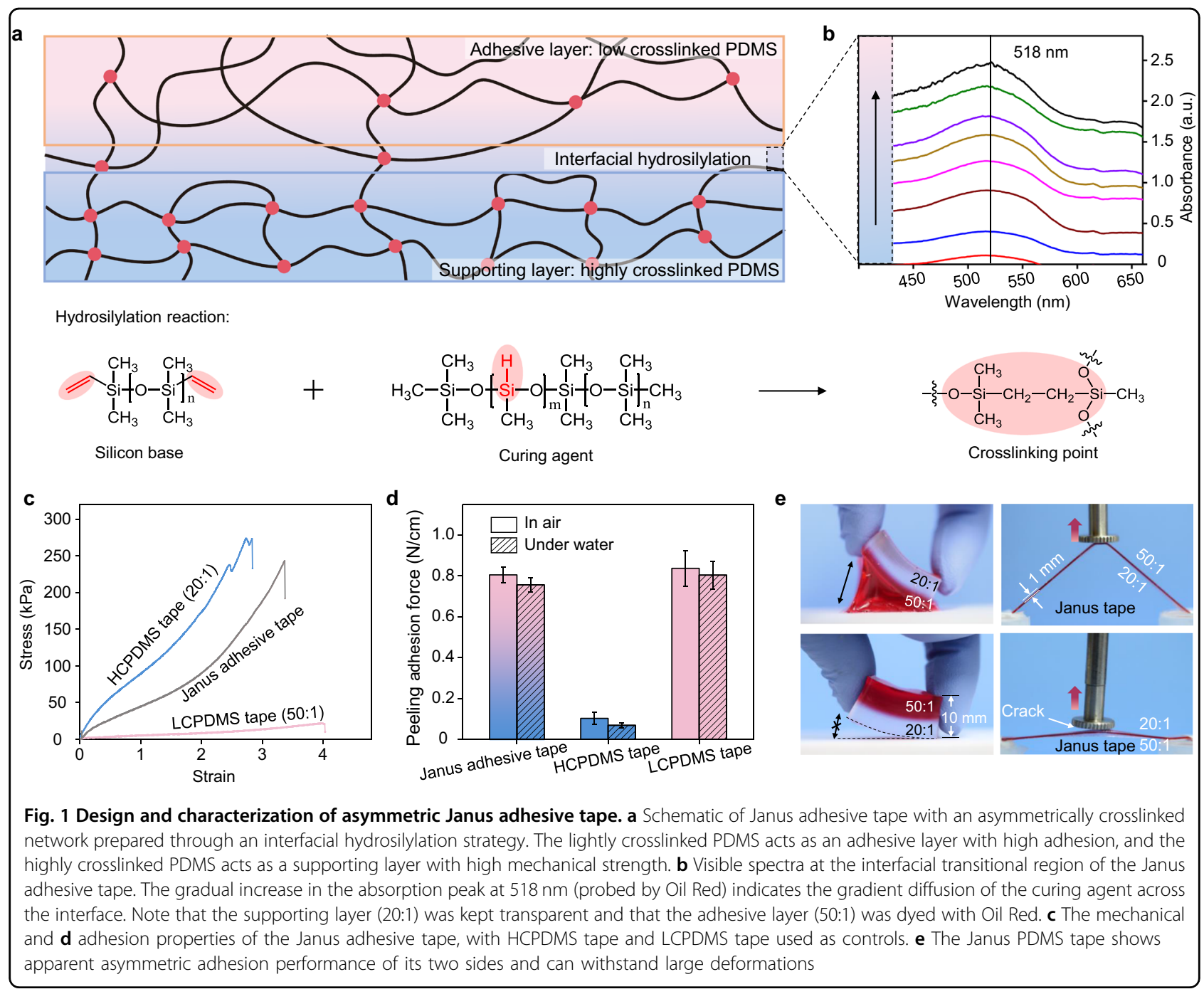

Herein, we present an interfacial hydrosilylation strategy to fabricate asymmetric Janus adhesive tape by curing lightly crosslinked PDMS on partially cured highly crosslinked PDMS. The lightly crosslinked side exhibiting high adhesion force acts as an adhesive layer, and the highly crosslinked side exhibiting high mechanical strength functions as a supporting layer (Fig. 1a). This asymmetric Janus adhesive tape simultaneously possesses good wet/dry adhesion performance and good mechanical properties and can adhere to different kinds of substrates. This study provides a new concept for designing nextgeneration adhesive materials with wet/dry amphibious adhesion properties.

\section{Experimental procedure}

\section{Materials}

PDMS-based elastomer Sylgard 184 (Dow Corning, United States), which consists of silicone base and a crosslinking agent.

\section{Procedures}

\section{Fabrication of Janus adhesive tape}

Different ratios of the silicone base and curing agent (Sylgard 184, Dow corning) were well mixed by a mechanical agitator in a plastic cup for $5 \mathrm{~min}$ at 500 rounds per min. The mixture was vacuumed in a vacuum pump for $30 \mathrm{~min}$ to remove air bubbles. Highly crosslinked PDMS (20:1) was cured at $80^{\circ} \mathrm{C}$ for 15 min to make it partially cured. Then, the same amount of lightly crosslinked PDMS precursor (50:1) (dyed with Oil Red) was poured on top of the precured 20:1 PDMS, and the two components were subsequently cured at $80^{\circ} \mathrm{C}$ for $1.5 \mathrm{~h}$ to make them completely joined together through an interfacial hydrosilylation reaction.

\section{Mechanical property testing}

The tensile test (stress, strain, and elongation at break) was performed with a dynamometer (M5-2; Mark-10 
Corporation). The stroke speed in the tensile test was $100 \mathrm{~mm} / \mathrm{min}$.

\section{Adhesion force testing}

The adhesion test of the Janus adhesive tape was performed with a dynamometer (M5-2; Mark-10 Corporation). The precursor mixtures were wiped on a polymeric support membrane (fluoroalkylsilane-modified polyethylene terephthalate film), and the samples for measurements were tailored to $10 \mathrm{~mm}$ wide, $1 \mathrm{~mm}$ thick, and $60 \mathrm{~mm}$ long. For the peeling adhesion force test, the peeling speed was $500 \mathrm{~mm} / \mathrm{min}$, and the maximum adhesion force was read while peeling off the rest of the sample from the substrate. All the tests were repeated at least three times.

\section{Surface modification of substrates}

The silanization reactions were performed at a temperature of $80^{\circ} \mathrm{C}$ under reduced pressure $(\approx 0.2 \mathrm{~atm})$ for $5 \mathrm{~h}$ by placing samples in a closed chamber. The silanes chosen to modify the substrates are (3-aminopropyl)trimethoxysilane, $\left(\mathrm{CH}_{3} \mathrm{O}\right)_{3} \mathrm{Si}\left(\mathrm{CH}_{2}\right)_{3} \mathrm{NH}_{2} \quad\left(\mathrm{C}_{3} \mathrm{NH}\right)$; ethyltrimethoxysilane, $\left(\mathrm{CH}_{3} \mathrm{O}\right)_{3} \mathrm{SiCH}_{2} \mathrm{CH}_{3}\left(\mathrm{C}_{2}\right)$; n-butyltrimethoxysilane, $\left(\mathrm{CH}_{3} \mathrm{O}\right)_{3}$ $\mathrm{Si}\left(\mathrm{CH}_{2}\right)_{3} \mathrm{CH}_{3}\left(\mathrm{C}_{4}\right)$; n-octyltrimethoxysilane, $\left(\mathrm{CH}_{3} \mathrm{O}\right)_{3} \mathrm{Si}\left(\mathrm{CH}_{2}\right)_{7}$

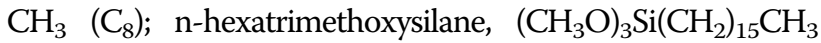
$\left(\mathrm{C}_{16}\right)$; and $1 \mathrm{H}, 1 \mathrm{H}, 2 \mathrm{H}, 2 \mathrm{H}$-perfluorododecyltrichlorosilane, $\mathrm{CF}_{3}\left(\mathrm{CF}_{2}\right)_{9}\left(\mathrm{CH}_{2}\right)_{2} \mathrm{SiCl}_{3}\left(\mathrm{C}_{10} \mathrm{~F}_{21}\right)$. The concentration of the silane was $5.0 \times 10^{-3} \mathrm{M}$.

\section{Surface topography characterization of the transferred polymer chains}

Silicon wafers tailored to the size of $1 \times 1 \mathrm{~cm}^{2}$ were cleaned by piranha solution (mixture of 7:3 (v/v) 98\% $\mathrm{H}_{2} \mathrm{SO}_{4}$ and $30 \% \mathrm{H}_{2} \mathrm{O}_{2}$ ) at $100^{\circ} \mathrm{C}$ for $1 \mathrm{~h}$, followed by thorough ultrasonication for $10 \mathrm{~min}$ in acetone and ethanol. Then, the silicon wafers were rinsed three times in Milli-Q water and blown dry with nitrogen gas. Next, the cleaned-washed silicon wafers were positioned under Milli-Q water, and three kinds of PDMS tapes, Janus adhesive tape, highly crosslinked PDMS (HCPDMS) tape, and lightly crosslinked PDMS (LCPDMS) tape, were vigorously and repeatedly attached to and detached from the surface of the silicon wafers three times. Subsequently, the silicon wafers were removed from the water and very gently blown dry with nitrogen gas. The surface topography of the transferred polymer chains was studied using an atomic force microscope (AFM, SPM-8000, Shimadzu). The AFM tip is N-type single-crystal silicon with a rectangular cantilever, and the force constant is $0.01-0.5 \mathrm{~N} / \mathrm{m}$. The same steps were carried out to inspect the surface residue with confocal laser scanning microscopy (CLSM) after immersing the attached/detached silicon wafers into rhodamine 110 solution.

\section{The contact state test between the Janus adhesive tape and a glass substrate}

In the CLSM observation experiment, Janus adhesive tape was labeled with 1,1'-dioctadecyl-3,3,3',3'-tetramethylindocarbocyanine perchlorate (DiI) ( $\geq 98 \%$; Aladdin) in red, and $\mathrm{H}_{2} \mathrm{O}$ was labeled with Rhodamine 110 $(\geq 98 \%$; Aladdin) in green. The concentration of the fluorescent dyes is $10 \mathrm{nM}$.

\section{Results and discussion}

As a widely used elastomer, PDMS often acts as a soft and sticky sealant in microfluidic ${ }^{33,34}$ and wearable electronic devices ${ }^{35,36}$ at a low crosslinking degree and as a hard and less sticky stamp for microscopic patterning ${ }^{37,38}$ at a high crosslinking degree. Its crosslinking degree can be regulated by changing the proportion of two precursors, silicone base and curing agent. In our design, we integrated LCPDMS $\left(w_{\text {silicone base }}: w_{\text {curing agent }}=50: 1\right)$ and HCPDMS $\quad\left(w_{\text {silicone }}\right.$ base $: w_{\text {curing }}$ agent $\left.=20: 1\right)$ together through an interfacial hydrosilylation reaction ${ }^{39}$. Briefly, we first prepared a partially crosslinked PDMS layer by curing a 20:1 mixture of silicone base and curing agent at $80^{\circ} \mathrm{C}$ for $15 \mathrm{~min}$. Then, we wiped a layer of $50: 1$ silicone base and curing agent on the precured 20:1 PDMS layer and cured them together at $80^{\circ} \mathrm{C}$ for $1.5 \mathrm{~h}$. The unreacted curing agent on the surface of the precured 20:1 PDMS layer reacted with the newly added silicon base of the 50:1 PDMS layer through a covalent hydrosilylation reaction. Across the interface, the crosslinking degree has a gradient transition from 20:1 to 50:1 because of the slow diffusion of the reactive curing agent ${ }^{40}$. By dying the 50:1 PDMS with Oil Red and keeping the 20:1 PDMS transparent, we traced the gradient diffusion of the curing agent with Oil Red as an indicator. A gradual increase in the characteristic peak at $518 \mathrm{~nm}$ (Oil Red) can indicate the gradient diffusion of the curing agent during interfacial hydrosilylation (Fig. 1b). The precuring time of the 20:1 PDMS is an important factor in the formation of Janus adhesive tape. If cured for a short time $(<10 \mathrm{~min})$, the 20:1 PDMS is still in mucus-like state and will irregularly fuse with the newly added 50:1 precursor mixture, which is thus unable to form the Janus tape. If cured for a long time (more than $30 \mathrm{~min}$ ), little residual curing agent from the 20:1 PDMS layer is left to further react with the newly added precursor mixture, resulting in a weakly bonded Janus tape whose two layers are easily separated (Fig. S1). With a suitable precuring time, the obtained Janus adhesive tape shows a high Young's modulus of $92.93 \mathrm{kPa}$ and strong wet adhesion of $0.76 \pm 0.03 \mathrm{~N} / \mathrm{cm}$ and dry adhesion of $0.80 \pm 0.04 \mathrm{~N} / \mathrm{cm}$ (Fig. 1c, d). In comparison, single-layer HCPDMS tape shows a high Young's modulus of $138.02 \mathrm{kPa}$ but weak wet adhesion of $0.07 \pm 0.01 \mathrm{~N} / \mathrm{cm}$ and dry adhesion of $0.10 \pm 0.03 \mathrm{~N} / \mathrm{cm}$ (note that PDMS becomes too hard when proportions of 
precursors higher than 20:1 are used). Single-layer LCPDMS tape shows strong wet adhesion of $0.80 \pm$ $0.07 \mathrm{~N} / \mathrm{cm}$ and dry adhesion of $0.84 \pm 0.09 \mathrm{~N} / \mathrm{cm}$ but a low Young's modulus of $4.93 \mathrm{kPa}$ (note that it is difficult to form films with proportions of PDMS precursors lower than 50:1). As shown in Fig. 1e, the Janus adhesive tape, which can be as thick as $10 \mathrm{~mm}$ or as thin as $1 \mathrm{~mm}$, shows apparent asymmetric adhesion performance on its two sides and can withstand large deformations. Therefore, we successfully fabricated Janus adhesive tape with good adhesion and mechanical properties through an interfacial hydrosilylation reaction.
We further demonstrated the adhesion and mechanical properties of the Janus adhesive tape simultaneously by a blocking experiment. The Janus adhesive tape (diameter of $30 \mathrm{~mm}$, thickness of $2 \mathrm{~mm}$ ) was fixed onto a hole (diameter of $15 \mathrm{~mm}$ ) on the underside of a $3 \mathrm{~L}$ beaker, and HCPDMS tape and LCPDMS tape samples with the same size were used as controls. As shown in Fig. 2a, the Janus adhesive tape can stop water leakage immediately after being pressed onto the hole. In comparison, the HCPDMS tape was washed away quickly from the hole once the hand left, which could be attributed to its weak wet adhesion $(0.07 \pm 0.01 \mathrm{~N} / \mathrm{cm})$. The LCPDMS tape can fix

a
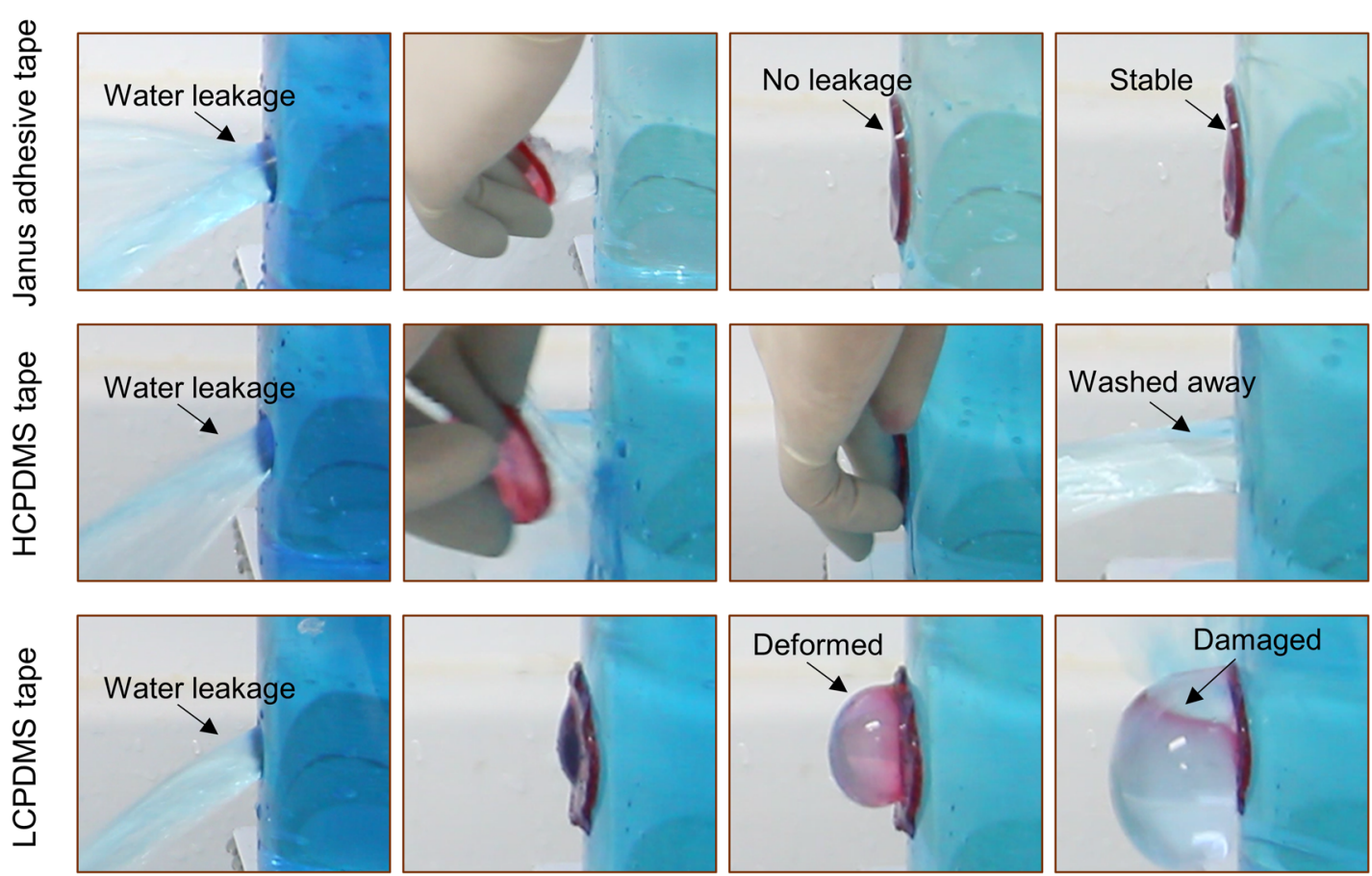

b
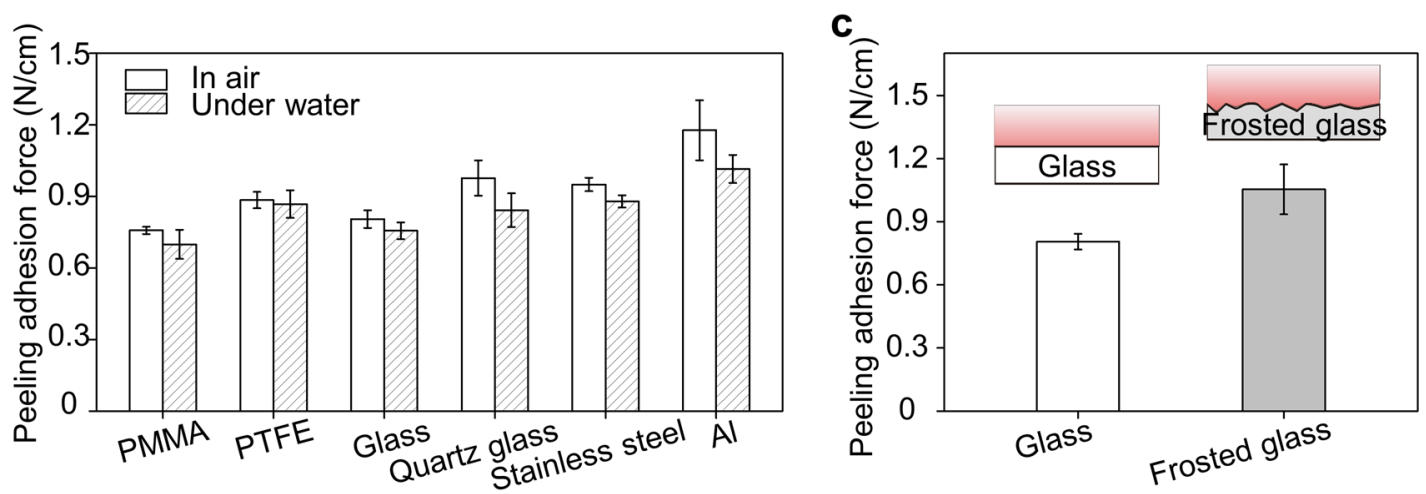

Fig. 2 Demonstration of the adhesion properties of the Janus PDMS tape. a Janus adhesive tape can attach firmly onto the wall of a $3 L$ beaker to stop water leakage. In comparison, the HCPDMS tape is easily washed away because of its poor adhesion properties, and the LCPDMS tape is easily damaged because of its poor mechanical properties. $\mathbf{b}$ The adhesion force of the Janus adhesive tape on various substrates, including polymers (PMMA and PTFE), inorganic nonmetallic materials (glass and quartz glass), and metallic materials (stainless steel and Al). c Comparison of adhesion force between Janus adhesive tape and smooth/frosted glass surfaces 

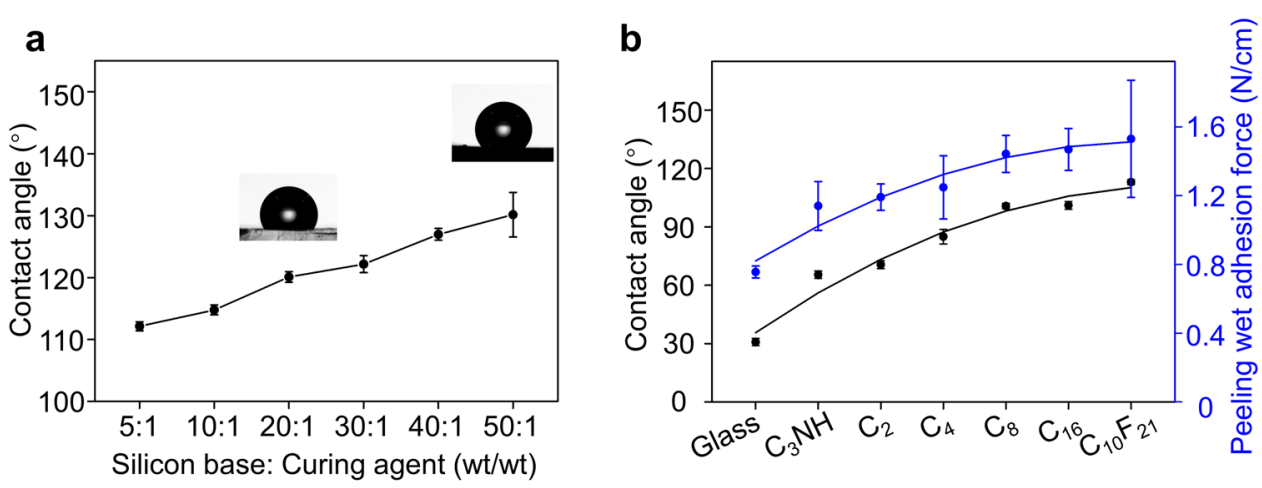

C

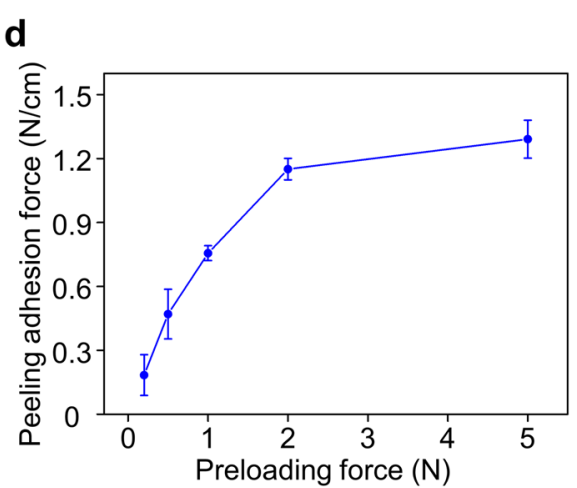

Fig. 3 Influence of surface wettability and preloading force on wet adhesion of the Janus adhesive tape. a The contact angle of PDMS samples with different crosslinking degrees. $\mathbf{b}$ Effect of surface wettability on wet adhesion to substrates modified with different silanes. $\mathbf{c}$ Confocal images of the underwater contact state between the Janus adhesive tape and glass substrate. $\mathbf{d}$ Effect of preloading force on wet adhesion of the Janus adhesive tape. The scale bar is $200 \mu \mathrm{m}$

on the hole in the beaker but was damaged in seconds, leading to leakage again. This failure mainly results from its low Young's modulus of $4.93 \mathrm{kPa}$ (Movie S1). In addition to flat glass substrates, this Janus adhesive tape can also adhere well to various solid surfaces, for instance, polymers (PMMA and PTFE), inorganic nonmetallic materials (quartz glass), and metallic materials (stainless steel and Al) (Fig. S2). The peeling adhesion force between Janus adhesive tape and these substrates under water was observed to range from $0.70 \pm 0.07$ to $1.10 \pm$ $0.06 \mathrm{~N} / \mathrm{cm}$, and similar results ranging from $0.76 \pm 0.02$ to $1.18 \pm 0.13 \mathrm{~N} / \mathrm{cm}$ were obtained in air (Fig. 2b). Moreover, the Janus adhesive tape shows stronger adhesion to rough substrates than flat substrates; e.g., the peeling adhesion force increases by $30.9 \%$ on frosted glass compared to flat glass (Fig. 2c). The low elastic modulus $(4.93 \mathrm{kPa})$ of the lightly crosslinked side attached to solid surfaces allows the Janus adhesive tape to deform easily to contact rough substrates and increase the contact area, resulting in stronger adhesion. These results verify that the Janus adhesive tape with good adhesion and mechanical properties can be applied to various solid substrates.

To explore the underwater adhesion mechanism of the Janus adhesive tape, we further investigated the influence of surface wettability on its wet adhesion properties. Since
PDMS with different crosslinking degrees (5:1, 10:1, 20:1, 30:1, 40:1, and 50:1) showed little change in hydrophobicity, with its air contact angle (CA) ranging from $112.1 \pm 0.7^{\circ}$ to $130.2 \pm 3.6^{\circ}$ (Fig. 3a), we tuned the surface wettability of substrates with $\mathrm{CA}$ values ranging from $30.8 \pm 1.9^{\circ}$ to $113.0 \pm 1.3^{\circ}$ by modifying naked glass substrates with a series of silanes with different functional groups and chain lengths. As the hydrophobicity of the substrates increases, the wet adhesion force between the Janus adhesive tape and substrates shows an upward trend from $0.70 \pm 0.07$ to $1.53 \pm 0.34 \mathrm{~N} / \mathrm{cm}$ (Fig. 3b). The underwater hydrophobic interaction has been observed at lengths up to hundred nanometers ${ }^{41,42}$. We speculate that when the hydrophobic Janus adhesive tape and the contacting surface, which is not strongly hydrophilic, are close enough, the hydrophobic interaction between them may expel the water layer, the existence of which is considered the main reason for the failure of wet adhesion ${ }^{43}$. To verify our speculation, CLSM was applied to further observe the contact state between the Janus adhesive tape and glass substrate under water on a microscale. Dioctadecyl-3,3,3',3'-tetramethylindocarbocyanine perchlorate (DiI), a red dye, was used to label the Janus adhesive tape, and rhodamine 110, a green dye, was used to label water. When the Janus adhesive tape was not applied, the water 
(green) was in direct contact with the glass substrate (black). However, when the tape was attached to the substrate under water, the Janus adhesive tape (red) directly contacted the substrate (black), and there was no residual water (green) between them, indicating that the water layer was expelled (Fig. 3c). When attaching Janus adhesive tape to substrates, the preloading force is an important factor in making the two surfaces close enough to maximize the hydrophobic interaction. Therefore, we then investigated the influence of preloading force on the wet peeling adhesion force of the Janus adhesive tape. As shown in Fig. 3d, the adhesion force showed an upward trend as the preloading force increased, which was in line with our speculation. The above results indicate that the hydrophobicity of Janus adhesive tape may facilitate wet adhesion by expelling the water layer with the assistance of preloading force.

After the water layer was driven away, the Janus adhesive tape could interact with substrates under water as it does in air. To better understand the wet adhesion performance of the Janus adhesive tape, we vigorously and repeatedly attached the Janus adhesive tape to and detached it from silicon wafers under water, with the HCPDMS tape and LCPDMS tape used as controls (Fig. S3). Atomic force microscopy (AFM) and CLSM were applied to characterize the surface topography changes of the silicon wafers on the nano- and microscale. As shown in Fig. 4a and b, after three cycles of attachment and detachment, AFM images showed that there were apparent topographic fluctuations on the surfaces treated

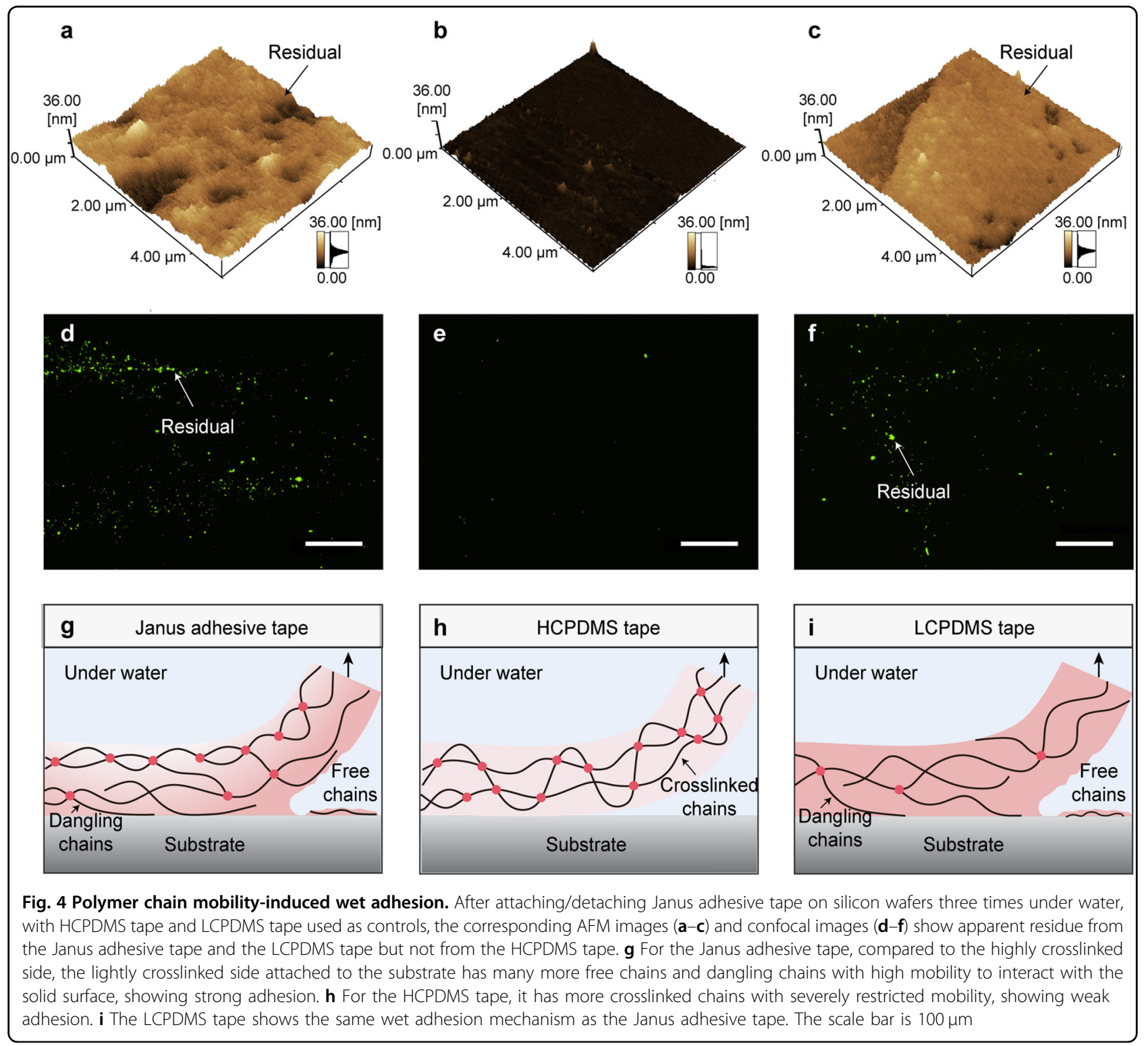


by the Janus adhesive tape and LCPDMS tape. Surface Raman spectra confirmed that the composition of the surface residue was PDMS (Fig. S4). In contrast, there was no apparent topographic fluctuation on the silicon surface treated with the HCPDMS tape (Fig. 4c). Subsequently, we visualized the surface residue on the microscale by CLSM by labeling the attachment-detachment-treated silicon wafers with rhodamine 110 solution (see Experimental section and Fig. S4). CLSM images showed similar results as AFM: there was apparent large-scale surface residue on the silicon wafers treated with the Janus adhesive tape and LCPDMS tape, in stark contrast with the wafers treated with the HCPDMS tape (Fig. 4d-f). In fact, if not pressed very hard, our Janus adhesive tape will leave little residue on the surface. Even if residue appears, in vitro cell culture ${ }^{44}$ and skin experiments ${ }^{45}$ have indicated the low cytotoxicity and good biocompatibility ${ }^{46}$ of residual PDMS. The different surface topographies on silicon wafers could be attributed to the different polymer chain mobilities of these three tapes (Fig. S5). For the Janus adhesive tape, compared to the highly crosslinked side, the lightly crosslinked side attached to solid surfaces has many more free chains and dangling chains with high mobility to interact with the solid surface. Moreover, some of the free chains can even be transferred to the solid surface and entangle with the nontransferred polymer chains upon the next contact process ${ }^{47}$, enabling strong wet adhesion (Fig. 4g). In comparison, the HCPDMS tape has more crosslinked chains with severely restricted mobility and thus exhibits weak wet adhesion (Fig. 4h). For the LCPDMS tape with more free chains and dangling chains, the wet adhesion mechanism was similar to that of the Janus adhesive tape (Fig. 4i). Furthermore, the excellent adhesion performance of the Janus adhesive tape can also be explained from the viewpoint of interfacial energy dissipation (Fig. S6). The lightly crosslinked side functions as an adhesive surface, and the highly/ lightly crosslinked PDMS layers act together as a dissipative matrix, which is tough and capable of dissipating energy effectively through hysteresis ${ }^{48,49}$. Therefore, the excellent adhesion performance of the Janus adhesive tape could be attributed to the synergistic effect of the two layers.

We further demonstrated the potential applications of our Janus adhesive tape in skin adhesion. The Janus adhesive tape shows a high transparency of almost $90 \%$ (Fig. 5a) and can be dyed different colors ranging from purple, pink, blue, and orange to red, which offers a variety of options in practice (Fig. 5b). Due to its high optical transparency, flexibility, nontoxicity, and

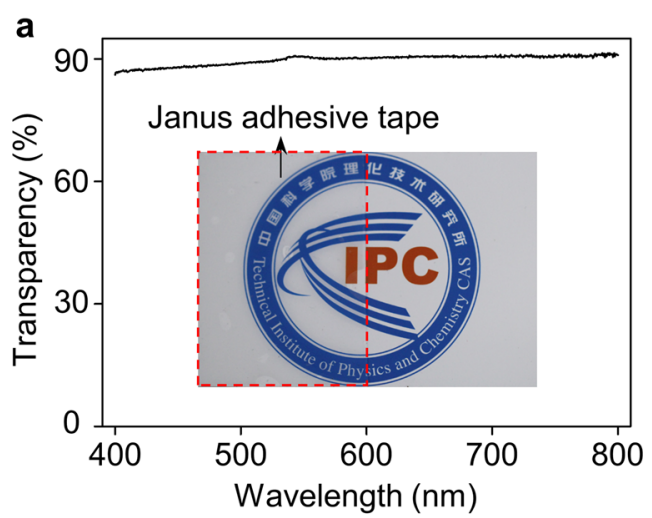

b

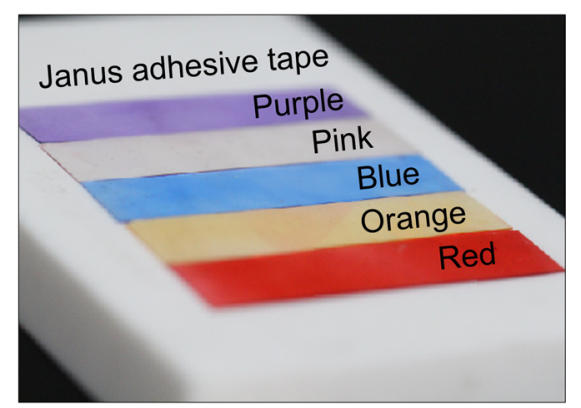

C
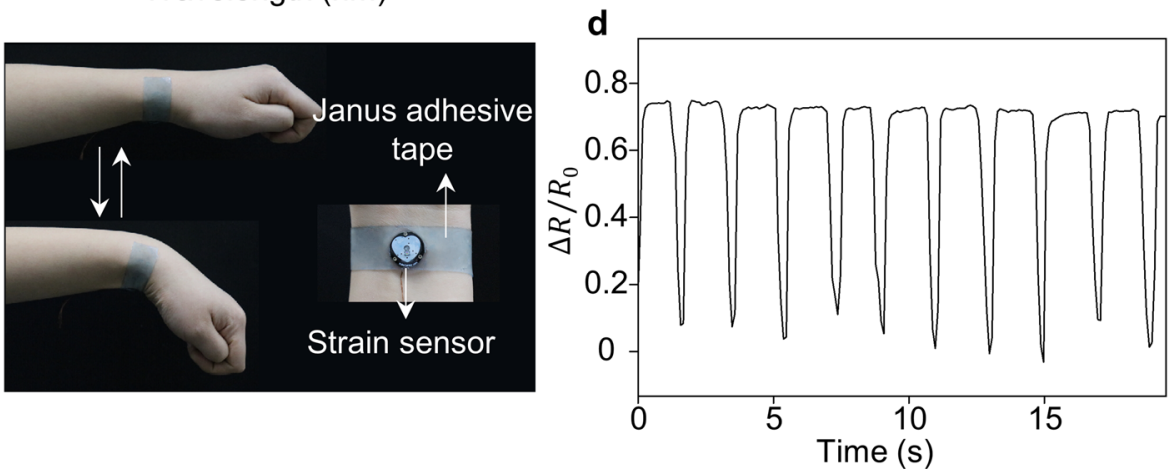

Fig. 5 Application of Janus adhesive tape in skin adhesion. a Janus adhesive tape shows a high transparency of almost 90\%. The inset image shows an optical photograph of the Janus adhesive tape. $\mathbf{b}$ The Janus adhesive tape can be dyed different colors. By attaching a strain sensor to the pulse point through Janus adhesive tape (c), the frequent bending motions of the wrist can be accurately monitored (d) 
biocompatibility, PDMS is often used in wearable electronic devices as an important fixing material ${ }^{35,36}$. However, the contradiction between the good mechanical and adhesion properties of PDMS restricts its practical application as a skin adhesive material. In contrast to our previous work $^{45}$, we solved this contradiction by integrating lightly and highly crosslinked PDMS with an interfacial hydrosilylation reaction and first revealed its underwater adhesion properties. Our Janus adhesive tape with simultaneous strong wet/dry adhesion and high strength may provide an alternative candidate material to replace traditional homogeneous tapes. To illustrate this possibility, we fabricated a force sensor sealed by dyed Janus adhesive tape. The sticky surface of the Janus adhesive tape provided conformal implementation of the force sensor on human skin (Fig. 5c). When the selfadhesive force sensor was applied to detect the bending motions of the wrist, the corresponding curves with good resolution and repeatability could be obtained (Fig. 5d). Therefore, this Janus adhesive tape shows strong, robust skin adhesion.

\section{Conclusion}

To conclude, we demonstrated an interfacial hydrosilylation strategy for fabricating Janus adhesive tape with an asymmetric crosslinked PDMS network and achieving effective wet/dry amphibious adhesion on diverse substrates. The wet adhesion performance of the Janus adhesive tape is attributed to the combination of the hydrophobic interaction and polymer chain mobility. This asymmetrical Janus adhesive tape with simultaneous good adhesive and mechanical properties solved the problem of the contradiction between good adhesion and mechanical properties in current homogeneous adhesive tapes, which is a key issue for achieving robust adhesion. We anticipate that this Janus adhesive tape can facilitate the use of underwater and skin adhesive materials and shows great application prospects in the field of wearable electronic devices.

\section{Acknowledgements}

This work was supported by the National Natural Science Foundation of China (21425314), the National Program for Special Support of Eminent Professionals and the Strategic Priority Research Program of the Chinese Academy of Sciences (XDA16020703).

\section{Author details}

${ }^{1}$ CAS Key Laboratory of Bioinspired Materials and Interfacial Science, CAS Center for Excellence in Nanoscience, Technical Institute of Physics and Chemistry, Chinese Academy of Sciences, Beijing 100190, P. R. China.

${ }^{2}$ University of Chinese Academy of Sciences, Beijing 100049, P. R. China. ${ }^{3}$ Department of Chemistry and Biological Engineering, University of Science and Technology Beijing, Beijing 100083, P. R. China. Institute for Stem Cell and Regeneration, Chinese Academy of Sciences, Beijing 100101, P. R. China

Conflict of interest

The authors declare that they have no conflict of interest.

\section{Publisher's note}

Springer Nature remains neutral with regard to jurisdictional claims in published maps and institutional affiliations.

Supplementary information is available for this paper at https://doi.org/ 10.1038/s41427-019-0150-x.

Received: 29 April 2019 Revised: 10 June 2019 Accepted: 14 June 2019. Published online: 6 September 2019

\section{References}

1. Oh, Y.-S. et al. Janus-faced, dual-conductive/chemically active battery separator membranes. Adv. Funct. Mater. 26, 7074-7083 (2016).

2. Zhang, Z. et al. Bioinspired heterogeneous ion pump membranes: unidirectional selective pumping and controllable gating properties stemming from asymmetric ionic group distribution. J. Am. Chem. Soc. 140, 1083-1090 (2018).

3. Zhang, Z. et al. Ultrathin and ion-selective Janus membranes for highperformance osmotic energy conversion. J. Am. Chem. Soc. 139, 8905-8914 (2017).

4. Liu, S., Wu, C., Hung, W.-S., Lu, X. \& Lee, K.-R. One-step constructed ultrathin Janus polyamide nanofilms with opposite charges for highly efficient nanofiltration. J. Mater. Chem. A 5, 22988-22996 (2017).

5. Zhang, F. et al. A monolithic hydro/organo macro copolymer actuator synthesized via interfacial copolymerization. NPG Asia Mater. 9, e380 (2017).

6. Zhao, Q. et al. An instant multi-responsive porous polymer actuator driven by solvent molecule sorption. Nat. Commun. 5, 4293-4300 (2014).

7. Zhang, $X$. et al. Photoactuators and motors based on carbon nanotubes with selective chirality distributions. Nat. Commun. 5, 2983-2990 (2014).

8. Yang, H. C., Hou, J., Chen, V. \& Xu, Z. K. Janus membranes: exploring fuality for advanced separation. Angew. Chem. Ed. 55, 13398-13407 (2016).

9. Yang, H. C. et al. Janus membranes: creating asymmetry for energy efficiency. Adv. Mater. 30, e1801495 (2018).

10. Shi, L., Liu, X., Wang, W., Jiang, L. \& Wang, S. A self-pumping dressing for draining excessive biofluid around wounds. Adv. Mater. 31, 1804187-1804195 (2018).

11. Zhang, Z. et al. Engineered asymmetric heterogeneous membrane: a concentration-gradient-driven energy harvesting device. J. Am. Chem. Soc. 137, 14765-14772 (2015).

12. Zhang, Z. et al. Asymmetric multifunctional heterogeneous membranes for $\mathrm{pH}$ - and temperature-cooperative smart ion transport modulation. Adv. Mater. 28, 9613-9619 (2016).

13. Yuk, H., Zhang, T., Parada, G. A., Liu, X. \& Zhao, X. Skin-inspired hydrogelelastomer hybrids with robust interfaces and functional microstructures. Nat. Commun. 7, 12028-12038 (2016).

14. Yu, Y. et al. Multifunctional "hydrogel skins" on diverse polymers with arbitrary shapes. Adv. Mater. 31, 1807101-1807109 (2018).

15. Yao, C. et al. Poly(N-isopropylacrylamide)-clay nanocomposite hydrogels with responsive bending property as temperature-controlled manipulators. Adv. Funct. Mater. 25, 2980-2991 (2015).

16. Ruibal, R. \& Ernst, V. The structure of the digital setae of lizards. J. Morphol. 117, 271-293 (1965).

17. Geim, A. K. et al. Microfabricated adhesive mimicking gecko foot-hair. Nat. Mater. 2, 461-463 (2003).

18. Autumn, K. et al. Adhesive force of a single gecko foot-hair. Nature 405, 681-685 (2000).

19. Gu, Z., Li, S., Zhang, F. \& Wang, S. Understanding surface adhesion in nature: a peeling model. Adv. Sci. 3, 1500327-1500339 (2016).

20. Waite, J. H. \& Tanzer, M. L. Polyphenolic substance of mytilus edulis: novel adhesive containing L-dopa and hydroxyproline. Science 212, 1038-1040 (1981).

21. Lee, H., Scherer, N. F. \& Messersmith, P. B. Single-molecule mechanics of mussel adhesion. Proc. Natl. Acad. Sci. USA 103, 12999-13003 (2006).

22. Maier, G. P., Rapp, M. V., Waite, J. H., Israelachvili, J. N. \& Butler, A. Adaptive synergy between catechol and lysine promotes wet adhesion by surface salt displacement. Science 349, 628-632 (2015).

23. Zhang, K., Zhang, F., Song, Y., Fan, J.-B. \& Wang, S. Recent progress of musselinspired underwater adhesives. Chin. J. Chem. 35, 820-829 (2017).

24. Swain, G. W., Griffith, J. R., Bultman, J. D. \& Vincent, H. L. The use of barnacle adhesion measurements for the field evaluation of non-toxic foul release surfaces. Biofouling 6, 105-114 (1992). 
25. Baik, S. et al. A wet-tolerant adhesive patch inspired by protuberances in suction cups of octopi. Nature 546, 396-400 (2017).

26. Sitti, M. \& Fearing, R. S. Synthetic gecko foot-hair micro/nano-structures as dry adhesives. J. Adhes. Sci. Technol. 17, 1055-1073 (2003).

27. Lee, $H$. et al. Octopus-inspired smart adhesive pads for transfer printing of semiconducting nanomembranes. Adv. Mater. 28, 7457-7465 (2016).

28. Statz, A. R., Meagher, R. J., Barron, A. E. \& Messersmith, P. B. New peptidomimetic polymers for antifouling surfaces. J. Am. Chem. Soc. 127, 7972-7973 (2005).

29. White, J. D. \& Wilker, J. J. Underwater bonding with charged polymer mimics of marine mussel adhesive proteins. Macromolecules 44, 5085-5088 (2011).

30. Matos-Perez, C. R., White, J. D. \& Wilker, J. J. Polymer composition and substrate influences on the adhesive bonding of a biomimetic, cross-linking polymer. J. Am. Chem. Soc. 134, 9498-9505 (2012).

31. Lee, H., Lee, B. P. \& Messersmith, P. B. A reversible wet/dry adhesive inspired by mussels and geckos. Nature 448, 338-341 (2007).

32. Ma, Y. et al. Remote control over underwater dynamic attachment/detachment and locomotion. Adv. Mater. 30, 1801595-1801602 (2018).

33. Wang, S. et al. Highly efficient capture of circulating tumor cells by using nanostructured silicon substrates with integrated chaotic micromixers. Angew. Chem. Ed. 50, 3084-3088 (2011).

34. Whitesides, G. M. The origins and the future of microfluidics. Nature $\mathbf{4 4 2}$ 368-373 (2006).

35. Wang, S., Oh, J. Y., Xu, J., Tran, H. \& Bao, Z. Skin-inspired electronics: an emerging paradigm. Acc. Chem. Res. 51, 1033-1045 (2018).

36. Wang, S. et al. Skin electronics from scalable fabrication of an intrinsically stretchable transistor array. Nature 555, 83-88 (2018).

37. Trau, M. et al. Microscopic patterning of orientated mesoscopic silica through guided growth. Nature 390, 674-676 (1997).
38. Xia, Y. \& Whitesides, G. M. Reduction in the size of features of patterned SAMs generated by microcontact printing with mechanical compression of the stamp. Adv. Mater. 7, 471-473 (1995).

39. Cai, L.-H. et al. Soft poly(dimethylsiloxane) elastomers from architecture-driven entanglement free design. Adv. Mater. 27, 5132-5140 (2015).

40. Eagan, J. M. et al. Combining polyethylene and polypropylene: enhanced performance with PE/iPP multiblock polymers. Science 355, 814-816 (2017).

41. Meyer, E. E., Rosenberg, K. J. \& Israelachvili, J. Recent progress in understanding hydrophobic interactions. Proc. Natl. Acad. Sci. USA 103 15739-15746 (2006).

42. Soltannia, B. \& Sameoto, D. Strong, reversible underwater adhesion via geckoinspired hydrophobic fibers. ACS Appl. Mater. Interfaces 6, 21995-22003 (2014).

43. Zhou, J., Anim-Danso, E., Zhang, Y., Zhou, Y. \& Dhinojwala, A. Interfacial water at polyurethane-sapphire interface. Langmuir 31, 12401-12407 (2015).

44. Fischer, S. C. L., Kruttwig, K., Bandmann, V., Hensel, R. \& Arzt, E. Adhesion and cellular compatibility of silicone-based skin adhesives. Macromol. Mater. Eng. 302, 1600526-1600526 (2017).

45. Gu, Z. et al. Skin adhesives with controlled adhesion by polymer chain mobility. ACS Appl. Mater. Interfaces 11, 1496-1502 (2019).

46. Sadtler, $\mathrm{K}$. et al. Clinical translation and immunological response of biomaterials in regenerative medicine. Nat. Rev. Mater. 1, 16040-16056 (2016).

47. Kroner, E., Maboudian, R. \& Arzt, E. Adhesion characteristics of PDMS surfaces during repeated pull-off force measurements. Adv. Eng. Mater. 12, 398-404 (2010).

48. Yuk, H., Zhang, T., Lin, S., Parada, G. A. \& Zhao, X. Tough bonding of hydrogels to diverse non-porous surfaces. Nat. Mater. 15, 190-196 (2016).

49. Li, J. et al. Tough adhesives for diverse wet surfaces. Science 357, 378-381 (2017). 